\title{
D-Dimer Unit
}

National Cancer Institute

\section{Source}

National Cancer Institute. D-Dimer Unit. NCI Thesaurus. Code C161494.

A unit of measure for the concentration of fibrin degradation products in a sample, calculated based upon the mass of D-dimers contained within that sample. 